\title{
Susceptibility to Ventricular Arrhythmias Resulting from Mutations in FKBP1B, PXDNL, and SCN9A Evaluated in hiPSC Cardiomyocytes
}

\author{
Hector Barajas-Martinez $\mathbb{D}^{1,2}$ Maya Smith, ${ }^{1}$ Dan Hu, ${ }^{1,3}$ Robert J. Goodrow, ${ }^{1}$ Colleen Puleo, ${ }^{1}$ \\ Can Hasdemir, ${ }^{4}$ Charles Antzelevitch, ${ }^{2,5}$ Ryan Pfeiffer, ${ }^{1}$ Jacqueline A. Treat, ${ }^{1}$ \\ and Jonathan M. Cordeiro ${ }^{1}{ }^{1}$ \\ ${ }^{1}$ Department of Experimental Cardiology, Masonic Medical Research Institute, Utica, NY, USA \\ ${ }^{2}$ Department of Cardiovascular Research, Lakenau Institute for Medical Research, Wynnewood, PA, USA \\ ${ }^{3}$ Department of Cardiology \& Cardiovascular Research Institute, Renmin Hospital of Wuhan University, Wuhan, China \\ ${ }^{4}$ Department of Cardiology, Ege University School of Medicine, Izmir, Turkey \\ ${ }^{5}$ Kimmel College of Medicine of Thomas Jefferson University, Philadelphia, PA, USA
}

Correspondence should be addressed to Hector Barajas-Martinez; barajasmartinezh@mlhs.org and Jonathan M. Cordeiro; jcordeiro@mmri.edu

Received 29 May 2020; Revised 29 July 2020; Accepted 11 August 2020; Published 1 September 2020

Academic Editor: Leonard M. Eisenberg

Copyright $\odot 2020$ Hector Barajas-Martinez et al. This is an open access article distributed under the Creative Commons Attribution License, which permits unrestricted use, distribution, and reproduction in any medium, provided the original work is properly cited.

Background. We report an inherited cardiac arrhythmia syndrome consisting of Brugada and Early Repolarization Syndrome associated with variants in SCN9A, PXDNL, and FKBP1B. The proband inherited the 3 mutations and exhibited palpitations and arrhythmia-mediated syncope, whereas the parents and sister, who carried one or two of the mutations, were asymptomatic. Methods and Results. We assessed the functional impact of these mutations in induced pluripotent stem cell cardiomyocytes (hiPSC-CMs) derived from the proband and an unaffected family member. Current and voltage clamp recordings, as well as confocal microscopy analysis of $\mathrm{Ca}^{2+}$ transients, were evaluated in hiPSC-CMs from the proband and compared these results with hiPSC-CMs from undiseased controls. Genetic analysis using next-generation DNA sequencing revealed heterozygous mutations in SCN9A, PXDNL, and FKBP1B in the proband. The proband displayed right bundle branch block and exhibited episodes of syncope. The father carried a mutation in $F K B P 1 B$, whereas the mother and sister carried the SCN9A mutation. None of the 3 family members screened developed cardiac events. Action potential recordings from control hiPSC-CM showed spontaneous activity and a low upstroke velocity. In contrast, the hiPSC-CM from the proband showed irregular spontaneous activity. Confocal microscopy of the hiPSC-CM of the proband revealed low fluorescence intensity $\mathrm{Ca}^{2+}$ transients that were episodic in nature. Patch-clamp measurements in hiPSC-CM showed no difference in $I_{\mathrm{Na}}$ but reduced $I_{\mathrm{Ca}}$ in the proband compared with control. Coexpression of PXDNL-R391Q with SCN5A-WT displayed lower $I_{\mathrm{Na}}$ density compared to PXDNLWT. In addition, coexpression of PXDNL-R391Q with KCND3-WT displayed significantly higher $I_{\text {to }}$ density compared to PXDNL-WT. Conclusion. SCN9A, PXDNL, and FKBP1B variants appeared to alter spontaneous activity in hiPSC-CM. Only the proband carrying all 3 mutations displayed the ERS/BrS phenotype, whereas one nor two mutations alone did not produce the clinical phenotype. Our results suggest a polygenic cause of the BrS/ERS arrhythmic phenotype due to mutations in these three gene variants caused a very significant loss of function of $I_{\mathrm{Na}}$ and $I_{\mathrm{Ca}}$ and gain of function of $I_{\mathrm{to}}$. 


\section{Introduction}

Cardiac myocytes derived from human-induced pluripotent stem cells (hiPSC-CM) can be used for a variety of applications such as regenerative therapy, cardiac safety pharmacology, and as models of human genetic disease. Specifically, patient-derived in vitro models can be used to study diseases of cardiac ion channels to determine how mutations in these channels alter the cardiac action potential [1]. Using this technology, hiPSC-CM can be generated in large quantities to elucidate the functional alterations resulting from the mutations. However, the disadvantage is that hiPSC-CM are immature, both electrophysiologically and morphologically $[2,3]$, suggesting that results obtained in hiPSC-CM may not translate to the adult phenotype of the disease.

It is well established that the development of cardiac arrhythmias is linked to mutations in genes that encode cardiac ion channels. These arrhythmias occur in both atria and ventricles and can appear in the absence of any structural defect. Long QT, Short QT, and Brugada Syndrome are all conditions linked to ion channelopathies [4]. For example, Short QT is characterized by QT intervals less than $330 \mathrm{~ms}$, and 8 different genes have been linked to Short QT syndrome [5]. Brugada Syndrome (BrS) is characterized by right bundle branch block and ST segment elevation in the right precordial leads (V1-V3) of an electrocardiogram [6], and 21 genes have been associated with BrS [7]. These inherited arrhythmia syndromes demonstrate that the majority of these genes encode cardiac ion channels resulting in a clear structurefunction alteration in the channel protein. In addition, other genes encode auxiliary subunits which do not conduct ions but are known to associate with ion channels and are important for channel gating and trafficking [8].

The manifestation of many arrhythmia syndromes is due to an imbalance in depolarizing and/or repolarizing currents during the cardiac action potential (AP). These imbalances result in a decrease in inward $I_{\mathrm{Na}}$ or $I_{\mathrm{Ca}}$ or an increase in outward $I_{\mathrm{K}}$ which would affect the morphology of the action potential resulting in the various syndromes [9, 10]. Recent studies have shown that mutations in genes not thought to be associated with cardiac ion channels can precipitate cardiac arrhythmias. For example, it is well documented that mutations in Ankyrin B are responsible for certain Long QT phenotypes [11, 12]. Moreover, it is clear that multiple genes may be involved in the development of cardiac arrhythmias highlighting the polygenic nature of these diseases $[13,14]$.

In this study, we identified a family that carried several mutations in genes not thought to be related to cardiac ion channels. One of these mutations was in the voltage-gated sodium channel alpha subunit 9 (SCN9A), a gene that encodes the neuronal sodium channel Nav1.7. A second mutation was found in a gene that encodes a peroxidasinlike protein $(P X D N L)$. The third mutation was found in FK506-binding protein $1 \mathrm{~B}(F K B P 1 B)$. FK506 is a commonly used immunosuppressant that binds to FK506-binding protein, and FKBP1B plays a role in heart failure and genetic forms of arrhythmias [15]. Interestingly, only the proband who inherited the 3 damaging mutations showed characteris- tics of Early Repolarization Syndrome (ERS), BrS, and right bundle branch block (RBBB) as well as numerous cardiac arrhythmias. We utilized hiPSC cardiomyocytes derived from the proband and an unaffected family member to systematically study the functional effects of these 3 mutations.

\section{Methods}

2.1. Subjects. IRB approval and informed consent were obtained from the family included in the study. The proband was identified following a visit to a physician due to episodes of palpitations and syncope, and ECG analysis initially revealed an ERS ECG. We studied three additional members of his family by genetic analysis: his sister and his parents.

2.2. Next-Generation Sequencing. An Ion Torrent Personal Genome Machine (PGM) was utilized to perform highthroughput sequencing (HTS) which gave us the capability to target and sequence 87 candidate genes at a time responsible for Brugada Syndrome [6, 8], Long QT [16], Short QT Syndrome [17, 18], and Early Repolarization Syndrome [10, 19, 20]. These candidate genes encode ion channels and other proteins including pumps, exchangers, calcium-handling proteins, gap junctional proteins, and structural proteins. They were selected based on their relative expression in human neuronal and cardiac tissue and their functional roles in generating action potentials and altering excitability.

The Ion Torrent PGM (Life Technologies, Carlsbad, CA) sequenced genomic DNA fragments generated through the use of Custom Ion Ampliseq 2.0 (Life Technologies, Carlsbad, CA). The Coding Exons as well as intron borders for our 87 genes of interest from human genomic DNA were amplified through a massive multiplex PCR approach. DNA libraries were prepared by attaching adapters to these fragments, as well as a unique molecular barcode for each sample. Library quality was assessed using the Qubit 2.0 Fluorometer and the dsDNA HS Assay (Life Technologies, Carlsbad, CA). An Ion Chef automation system then performed emulsion PCR, enrichment, and chip loading, followed by direct sequencing with the Ion Torrent PGM.

2.3. Mutation Confirmation. All rare variations and mutations uncovered were confirmed using gold standard Sanger sequencing. PCR products were purified with a commercial enzyme (ExoSAP-IT, USB, Cleveland, OH) and directly sequenced from both directions using Big Dye Terminator 3.1 chemistry on an Applied Biosystems 3730 DNA Analyzer (Life Technologies, Carlsbad, CA).

2.4. Bioinformatics Analysis. After signal processing and basecalling, the Ion Torrent Suite software was used to map the sequencing reads to the DNA reference sequence [hg19] and identify variants through the VariantCaller plugin as well as the IonReporter analysis tool. Run Quality was assessed using the Coverage Analysis plugin which reports average depth of coverage, uniformity of coverage, and percent on target as well as other metrics. IonReporter compares all variations identified against NCBI's dbSNP to rule out common SNP's, as well as the 1000 genomes project and Exome Sequencing Project (ESP) to get published frequencies. 
Variants of interest were then flagged for verification through Sanger sequencing. Mutations and rare variants are analyzed using several pathogenicity prediction tools such as PolyPhen2, SIFT, and Grantham. When available, family members are sequenced for these mutations and rare variants to analyze the penetrance and establish a genotype-phenotype correlation.

2.5. Generation of hiPSC-CM. The control and patientderived human iPS cells were reprogrammed from fibroblasts with Oct4, Sox2, Lin28, and Nanog. A directed differentiation protocol to derive cardiomyocytes using serum-free, chemically defined media supplemented with CHIR99021, IWP2, Activin A, and KY0211 in stage specific manner, as previously described [21]. This protocol yielded contractile clusters by days 9 to 12 postdifferentiation. Monolayers ranging between 20 and 50 days of maturity were plated on matrigel-coated dishes and maintained with RPMI B27+ until use.

2.6. Fluorescence Imaging. Fluo 4-AM was used for this study. Fluo 4-AM dissolved in 20\% F-127 pluronic in dimethyl sulfoxide (DMSO) for $20 \mathrm{~min}$ at room temperature as previously described $[22,23]$. Confocal experiments were performed with an Olympus Fluoview laser-scanning confocal microscope. Fluo 4-loaded iPSC-CMs were placed in a perfusion chamber and excited at $488 \mathrm{~nm}$ using an argon laser, and fluorescence emission was detected via a $520 \mathrm{~nm}$ band-pass filter and photomultiplier tube. Confocal images were acquired with the Fluoview acquisition software program, and spontaneous activity was recorded on a personal computer for later analysis. Images acquired with Fluoview acquisition software were analyzed with ImageJ and Fluoview analysis software.

2.7. Cell Transfection/Mutagenesis. Site-directed mutagenesis was performed using QuikChange (Stratagene, LaJolla, CA) on full-length human wild type (WT) PXDNL cDNA cloned in pcDNA3. TSA201 cells were grown in DMEM with Glutamax supplemented with $10 \%$ FBS in $35 \mathrm{~mm}$ culture dishes and placed in a $5 \% \mathrm{CO}_{2}$ incubator at $37^{\circ} \mathrm{C}$. To determine whether the R391Q mutation in PXDNL altered the biophysical characteristics of $I_{\mathrm{Na}}$ or $I_{\text {to }}$, TSA201 cells were cotransfected with a combination of mutant or WT PXDNL. To assess the effect on $I_{\mathrm{Na}}$, cells were cotransfected using FuGene6 (Roche Diagnostics, Indianapolis, IN) with a $1: 1$ molar ratio of WT human SCN5A, and WT or R391Q mutant PXDNL [24]. To assess the effect on $I_{\text {to }}$, cells were cotransfected using FuGene6 (Roche Diagnostics, Indianapolis, IN) with a 1:1 molar ratio of WT human KCND3, and WT or R391Q mutant PXDNL. In addition, $0.20 \mu \mathrm{g}$ of enhanced green fluorescent protein cDNA was added to the transfection mixture. Cells displaying fluorescence $48-72 \mathrm{~h}$ after transfection were used for electrophysiological study.

2.8. Electrophysiology. Sharp microelectrodes (40-60 M $\Omega$ ) filled with $2.7 \mathrm{M} \mathrm{KCl}$ were used to record APs from spontaneously beating monolayers, as previously described [25]. Monolayers were superfused with HEPES-Tyrode's solution of the following composition (in $\mathrm{mM}$ ): $\mathrm{NaCl} \mathrm{140,} \mathrm{KCl} 4$,
$\mathrm{MgCl}_{2}$ 1, HEPES 10, D-Glucose 10 , and $\mathrm{CaCl}_{2} 2 ; \mathrm{pH}$ was adjusted to 7.4 with $\mathrm{NaOH}$. The microelectrodes were connected to an MultiClamp 700B amplifier (Molecular Devices, Foster City, CA, USA) operating in current clamp mode. All signals were digitized (sampling rate $=50 \mathrm{kHz}$ ), stored on a computer, and analyzed using pClamp9 acquisition suite (Molecular Devices, Foster City, CA).

Voltage clamp recordings were made using patch pipettes fabricated from borosilicate glass capillaries (1.5 mm O.D., Fisher Scientific, Pittsburg, PA). The pipettes were pulled using a gravity puller (Model PP-830, Narashige Corp.) and filled with pipette solution of the following composition (mM): for $I_{\mathrm{Ca}}$ recordings, the patch pipette contained (in $\mathrm{mM}$ ) $\mathrm{CsCl} 120, \mathrm{MgCl}_{2}$ 1.0, EGTA 10, MgATP 5, HEPES, and $\mathrm{CaCl} 25, \mathrm{pH}=7.2$ with $\mathrm{CsOH}$ [26]. For $I_{\mathrm{Na}}$ recordings in hiPSC-CMs, the external solution contained (in $\mathrm{mM}$ ) $\mathrm{N}$ Methyl D-Glucamine 105, $\mathrm{NaCl} 40, \mathrm{CaCl}_{2} 2.0, \mathrm{MgCl}_{2} 1.0$, glucose 10 , HEPES free acid $10, \mathrm{CdCl}_{2} 0.3, \mathrm{pH}$ adjusted to 7.4 with $\mathrm{NaOH} / \mathrm{HCl}$. For $I_{\mathrm{Na}}$ recordings in transfected TSA cells, the perfusion solution contained (in $\mathrm{mM}$ ) $\mathrm{NaCl} 130$, $\mathrm{KCl} 5, \mathrm{CaCl}_{2} 1.8, \mathrm{MgCl}_{2} 1.0$, sodium acetate 2.8, HEPES 10, glucose $10, \mathrm{pH} 7.4$ with $\mathrm{NaOH}$. The pipette solution contained (in $\mathrm{mM}$ ) $\mathrm{NaCl} 15, \mathrm{CsF} 120, \mathrm{MgCl}_{2} 1, \mathrm{KCl} 5$, HEPES 10, $\mathrm{Na}_{2} \mathrm{ATP} 4$, and EGTA 10, pH adjusted to 7.2 with $\mathrm{CsOH}$ $[25,27]$. For hiPSC-CM recordings, peak $I_{\mathrm{Na}}$ was dramatically reduced in the low extracellular $\mathrm{Na}^{+}$solution to ensure adequate voltage control, as gauged by the slope of a Boltzmann fit to the steady-state activation curve [28]. Current signals were recorded using a MultiClamp 700A amplifier (Axon Instruments Inc., Foster City, CA), and series resistance errors were reduced by about $60-70 \%$ with electronic compensation. Signals were acquired at $20-50 \mathrm{kHz}$ (Digidata 1322, Axon Instruments) and analyzed with a microcomputer running pClamp 9 software (Axon Instruments, Foster City, CA). All recordings were made at $37^{\circ} \mathrm{C}$ with the exception of $\mathrm{I}_{\mathrm{Na}}$ which was performed at room temperature.

2.9. Statistics and Data Analysis. $\mathrm{Ca}^{2+}$ transient and electrophysiological data are presented as Mean \pm SEM, and statistical comparisons were made using ANOVA followed by a Student-Newman-Kuels, or Student's $t$-test, as appropriate. Significance was determined at $p<0.05$.

\section{Results}

The proband (MMRL1126) was a male who initially presented with palpitations and syncope. ECG analysis of the patient revealed a slurring at the end of the QRS complex and ST-segment elevation leading to an initial diagnosis of ERS (Figure 1(a)). The patient was given dobutamine which resulted in sustained monomorphic VT and syncope originating from septal RVOT which persisted after dobutamine infusion was discontinued (Figure 1(b)). RBBBc was developed in the proband after radiofrequency catheter ablation in septal wall of RVOT (Figure 1(c)). Patient was challenged with ajmaline resulting in unmasking of BrS ECG phenotype (Figure 1(d)).

We performed NextGen sequencing of the index patient as well as the sister and the parents. Genetic analysis of the 


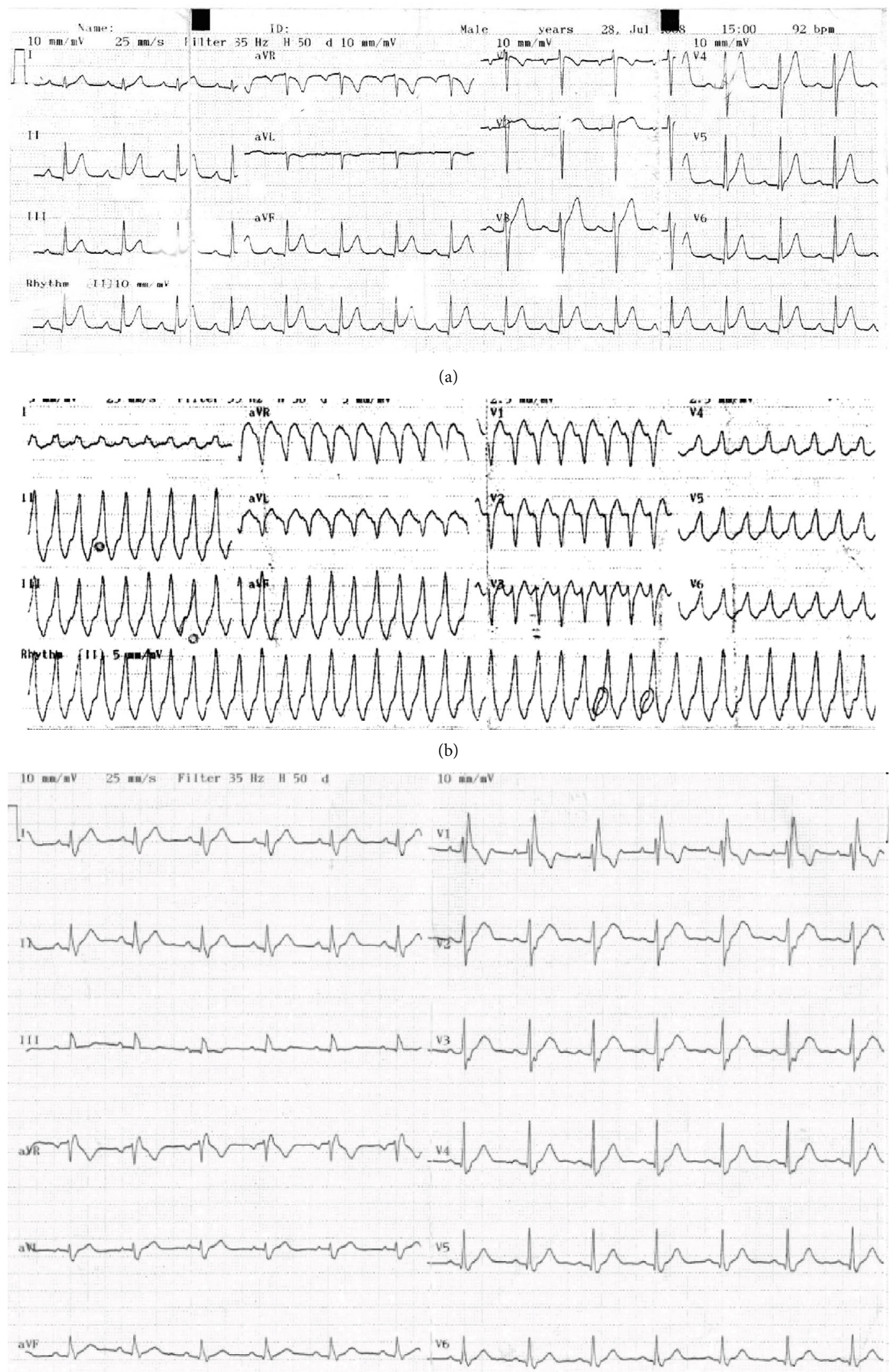

(c)

FIgUre 1: Continued. 


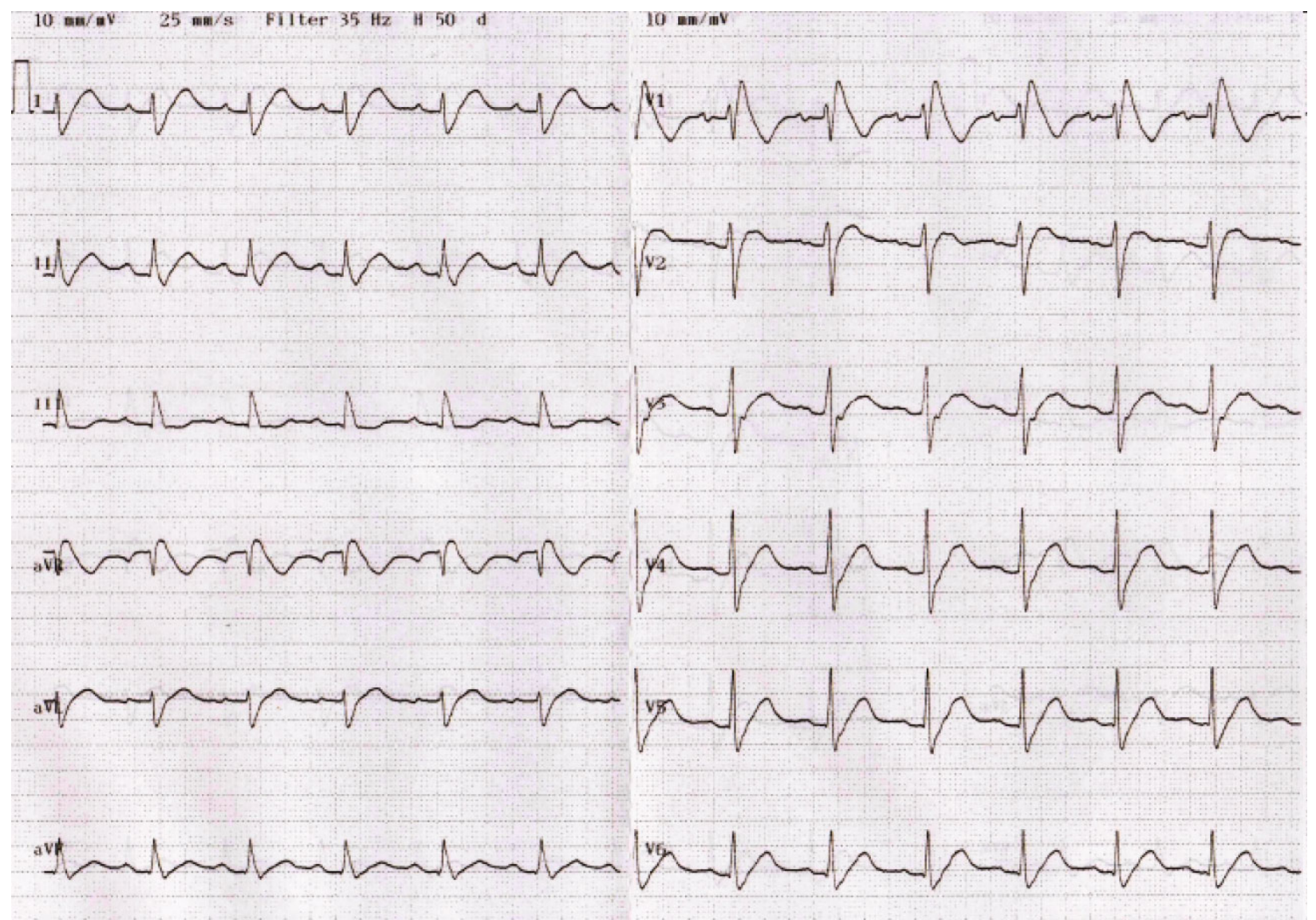

(d)

FIGURE 1: (a) ECG analysis of patient MMRL 1126 showing early repolarization phenotype. A prominent ST segment elevation and slurring of the QRS complex is apparent in several leads. (b) Infusion of dobutamine caused the appearance of monomorphic VT in MMRL1126 which persisted after dobutamine was discontinued. (c) The arrhythmogenic focus was ablated and patient subsequently exhibited RBBB apparent in lead V1. (d) Patient was challenged with ajmaline resulting in unmasking of BrS.

index patient revealed three heterozygous exonic mutations, one in SCN9A, another in PXDNL, and the third in the $F K B P 1 b$ gene (Figure 2). The SCN9A gene was mutated from a $\mathrm{T}$ to $\mathrm{C}$ substitution at position 3253 in exon 17, resulting in an amino acid change from serine to proline at position 1085 (S1085P). The FKBP1b gene was mutated to a $\mathrm{C}$ deletion at location 137 in exon 3 resulting in complexed amino acid changes. The amino acid change was proline to leucine at position 46; the next 22 amino acids were frame shifted culminating in a stop codon 22 amino acids downstream (P46L fsx22). The PXDNL gene was mutated from a $\mathrm{G}$ to $\mathrm{A}$ at position 1172 in exon 14 resulting in an amino acid change from an arginine to a glutamine at position 391 (R391Q). Mutations and polymorphisms of the proband are shown in Table 1 and Figure 3.

Pedigree analysis of the family showed that the mother of the proband carried both the SCN9A and PXDNL mutations, whereas the father carried the FKBP1b gene mutation, and both were found to be asymptomatic. The sister inherited only the SCN9A gene mutation and was also asymptomatic (Figure 3).

Patient MMRL 1126 and unaffected family member MMRL 1239 carried a mutation in FKBP1b gene. The $F K B P 1 b$ gene encodes for a binding protein, calstabin 2, which is closely associated with the cardiac ryanodine receptor (RYR2) and is believed to play a role in the regulation of RYR2 open probability. Mutations in the FKBP1b gene may affect $\mathrm{Ca}^{2+}$ release from the SR resulting in alterations in the $\mathrm{Ca}^{2+}$ transient as well as indirectly affecting action potential waveform and duration. As an initial basis of comparison, we recorded calcium transients in normal (WT) hiPSC-CMs and hiPSC-CMs derived from affected patient MMRL1126 and unaffected family member MMRL 1239 (see Figure 3). Cells were loaded with fluo-4 AM, and $\mathrm{Ca}^{2+}$ transients from spontaneously beating cells were recorded (Figure 4(a)). Compared to control and MMRL 1239 monolayers, $\mathrm{Ca}^{2+}$ transients from patient 1126 showed slower spontaneous activity and had a lower fluorescence intensity (Table 2). In addition, episodic or intermittent spontaneous activity was noted in a number of recordings (34/93). The lower fluorescence intensity suggests that there is less intracellular calcium present in MMRL1126 cells during the course of an action potential. Interestingly, even though MMRL 1239 carried the mutation in FKBP1b, the $\mathrm{Ca}^{2+}$ showed different characteristics compared to MMRL 1126 and seemed to resemble WT hiPSC-CM although some episodic transients were observed. 


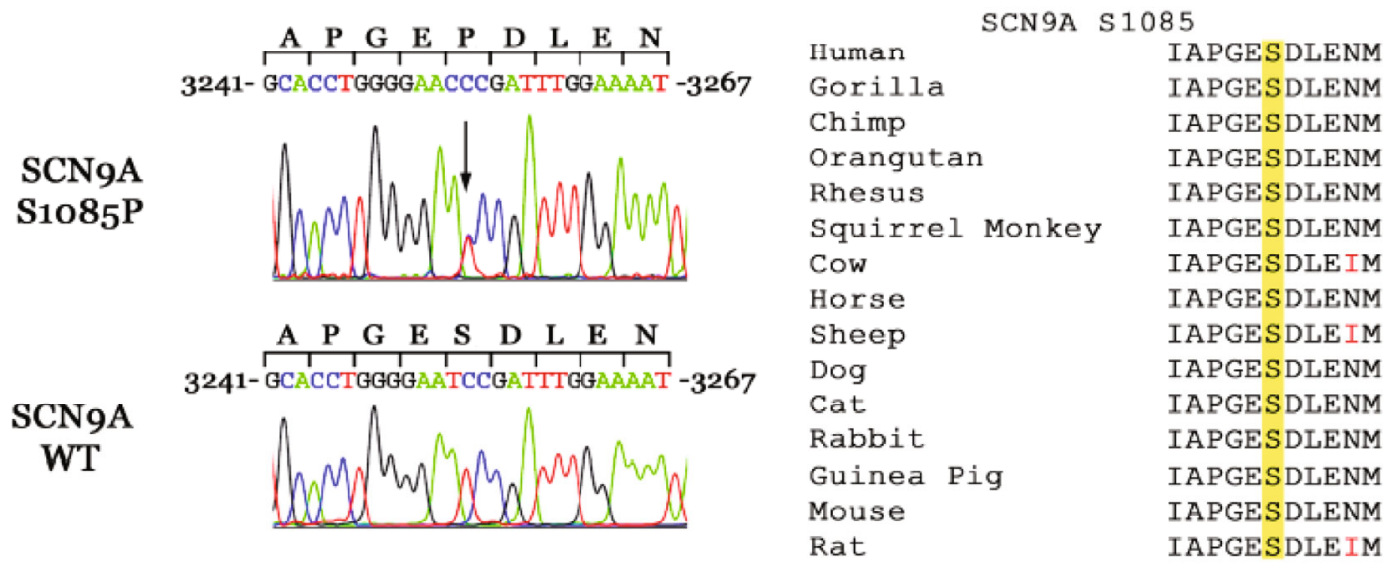

FIGURE 2: DNA sequencing analysis of SCN9A and FKBP1b. The FKBP1b gene was mutated to a C deletion at location 137 in exon 3 resulting in complexed amino acid changes. The amino acid change was proline to leucine at position 46; the next 22 amino acids were framed shifted culminating in a stop codon 22 amino acides downstream (P46L fsx22). The SCN9A gene was mutated from a T to C substitution at position 3253 in exon 17, resulting in an amino acid change from serine to proline at position 1085 (S1085P). The PXDNL gene was mutated from a G to $\mathrm{A}$ at position 1172 in exon 14, resulting in an amino acid change from arginine to glutamine at position 391 (R391Q). Mutations in SCN9A and FKBP $1 b$ are highly conserved across the various species. PXDNL is only expressed in certain mammals including primates. 
TABLE 1: MMRL1126 87 gene panel results.

\begin{tabular}{|c|c|c|c|c|c|c|c|}
\hline \# locus & Gene & Exon & Protein & Coding & Sift & Polyphen & dbsnp \\
\hline chr2:24283733 & FKBP1b & 3 & p.Lys48fs & c.136delC & VUS & VUS & rs200092869 \\
\hline chr7:91714911 & AKAP9 & 41 & NEGATIVE & & & & rs 1063242 \\
\hline chr2:167128974 & SCN9A & 17 & p.Ser1085Pro & c. $3253 \mathrm{~T}>\mathrm{C}$ & Damaging & Possibly damaging & rs12720442 \\
\hline chr8:52366156 & PXDNL & 14 & p.Arg391Gln & c. $1172 \mathrm{G}>\mathrm{A}$ & VUS & VUS & rs79394014 \\
\hline chr2:167262238 & SCN7A & 25 & NEGATIVE & & & & rs80098689 \\
\hline chr2:166900411 & SCN1A & 11 & NEGATIVE & & & & rs121918769 \\
\hline
\end{tabular}
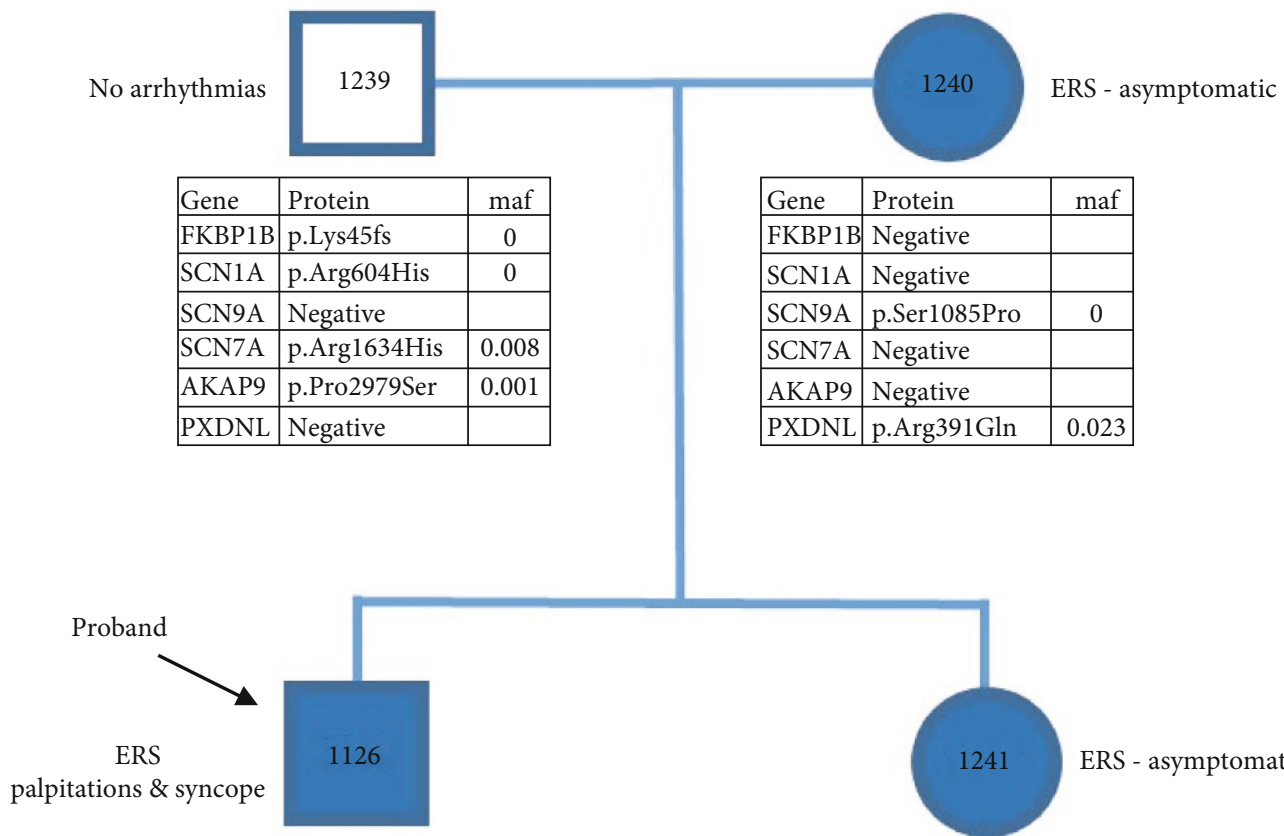

\begin{tabular}{|l|l|c|}
\hline Gene & Protein & maf \\
\hline FKBP1B & p.Lys48fs & 0 \\
\hline SCN1A & Negative & \\
\hline SCN9A & p.Ser1085Pro & 0 \\
\hline SCN7A & Negative & \\
\hline AKAP9 & Negative & \\
\hline PXDNL & p.Arg391Gln & 0.023 \\
\hline
\end{tabular}

\begin{tabular}{|l|l|c|}
\hline Gene & Protein & maf \\
\hline FKBP1B & Negative & \\
\hline SCN1A & Negative & \\
\hline SCN9A & p.Ser1085Pro & 0 \\
\hline SCN7A & Negative & \\
\hline AKAP9 & p.Pro2979Ser & 0.001 \\
\hline PXDNL & Negative & \\
\hline
\end{tabular}

FIGURE 3: Pedigree of the proband (denoted with arrow) and family. Genetic analysis revealed that each parent carried different heterozygous mutation(s). The father (1239) carried the FKBP1b mutation, whereas the mother (1240) carried the PXDNL and SCN9A mutations. The sister of the proband (1241) only inherited the mutation in SCN9A. Both parents and the sister were asymptomatic.

Since MMRL 1126 showed clinical symptoms, and hiPSCCMs derived from this patient exhibited abnormal spontaneous activity, we systematically evaluated the mechanism for these differences. We further evaluated hiPSC-CM derived from MMRL 1126 by recording action potentials from WT and MMRL1126 hiPSC-CMs. Cells were plated at high density to form monolayers, and a detailed analysis of their electrophysiological characteristics was performed. Action potentials recorded from control monolayers were spontaneously active, exhibited a slow upstroke velocity, and no phase 1 repolarization. Recordings from hiPSC-CMs derived from the proband also showed spontaneous activity (Figure 5). However, the spontaneous activity was episodic, and brief periods of no spontaneous activity was also observed (8/13 monolayers).
In monolayers derived from the proband, MDP was more depolarized $(-57.8 \pm 2.1$ vs. $-68.7 \pm 1.1 \mathrm{mV} ; p<0.05)$, and $V_{\max }$ was lower $(17.3 \pm 1.6$ vs. $38.5 \pm 2.4 \mathrm{~V} / \mathrm{s} ; p<0.05)$ compared to controls. In addition, $\mathrm{APD}_{50}$ and $\mathrm{APD}_{90}$ were briefer than that observed in WT hiPCS-CMs (Table 3).

Genetic analysis revealed that the proband carried 3 mutations, one of which was in PXDNL. The PXDNL gene encodes for a peroxidasin-like protein which is expressed in the heart. Previous immunocytochemical studies of PXDNL protein demonstrated that the protein product is highly expressed on the membrane of human ventricular cells, especially at the intercalated discs [29]. In prior studies from our labs, we noted a similar expression pattern of $\mathrm{Na}^{+}$channels in ventricular cells [30] suggesting that the PXDNL protein may 


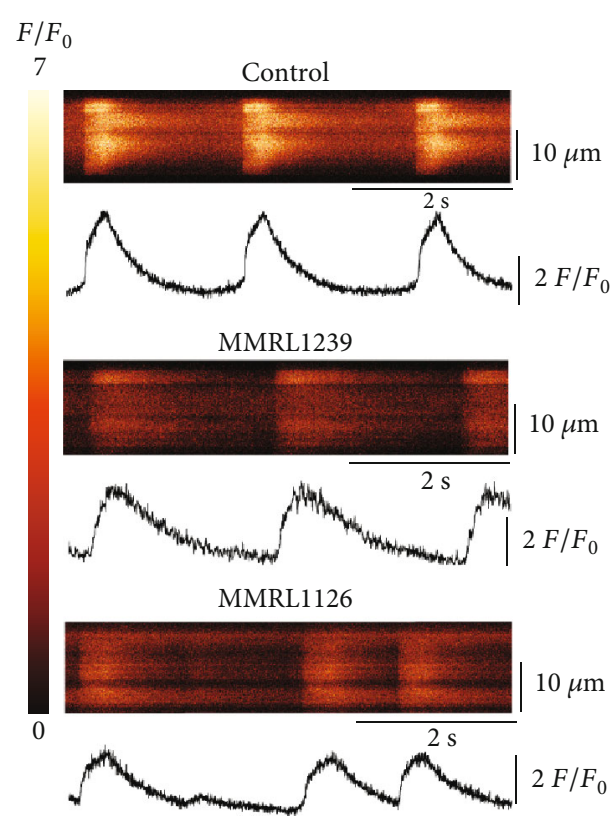

FIgURe 4: Line scans recorded from WT, MMRL 1239, and MMRL1126 hiPSC-CMs showing spontaneous $\mathrm{Ca}^{2+}$ transients in all hiPSC-CMs. However, fluorescence intensity was much lower in MMRL 1126, and spontaneous activity was episodic in nature.

colocalize with $\mathrm{Na}^{+}$channels. This potential interaction may result in an alteration in the size of the $\mathrm{Na}^{+}$current, contributing to the lower $V_{\max }$ measured in the hiPSC-CMs of the proband (Table 3). We next measured peak $I_{\mathrm{Na}}$ in WT and MMRL1126 hiPSC-CMs. Representative traces are shown in Figures 6(a) and 6(b). $I_{\mathrm{Na}}$ was activated by a series of depolarizing pulses from -80 to $+30 \mathrm{mV}$ in $5 \mathrm{mV}$ increments. Analysis of the current-voltage relation showed that peak $I_{\mathrm{Na}}$ was $-72.5 \pm 6.5 \mathrm{pA} / \mathrm{pF}$ for $\mathrm{WT}$ and $-80.9 \pm 7.0$ for MMRL1126 at $-35 \mathrm{mV}$ (Figure 6(c), $p=$ N.S.). Steady state activation was not significantly different between WT and MMRL1126 (Figure 6(d)). Although $I_{\mathrm{Na}}$ current density was not significantly different between WT and MMRL1126, the lower $V_{\max }$ observed in hiPSC-CMs from MMRL 1126 could possibly be due to a shift in $\mathrm{Na}^{+}$channel availability. We next evaluated steady-state inactivation between the two cell types. Peak current after a $500 \mathrm{~ms}$ prepulse was normalized to the maximum current and plotted as a function of the prepulse voltage. A Boltzman function was then fit to the data. Figures 6(e) and 6(f) shows representative traces recorded from a WT and MMRL1126 hiPSC-CM. The midinactivation potential was $-79.3 \pm 0.12 \mathrm{mV}$ for $\mathrm{WT}$ and $-80.0 \pm 0.16$ for MMRL1126 ( $p=$ N.S.) These results demonstrate that the difference in $V_{\max }$ between WT and MMRL1126 was not due to a shift in steady-state inactivation (Figure 6(g)) and suggest that the difference in $V_{\max }$ between WT and MMRL1126 is likely due to the more depolarized MDP in the proband.

The SCN9A gene encodes Nav1.7 sodium channels which are found mainly in nerve cells and transmit pain signals. Interestingly, most reported mutations in Nav1.7 result in either insensitivity to pain (due to a loss of function in this channel) [31] or a hypersensitivity to pain (due to a gain of function in this channel) [32]. Previous studies have shown the presence and functional role for neuronal or "brain type" $\mathrm{Na}^{+}$channels in the heart although Nav1.7 was not probed in those studies [30, 33, 34]. We first used WT hiPSC-CMs and probed for both $S C N 5 A$ and $S C N 9 A$ to determine the level of transcript. RT-PCR analysis showed that $S C N 5 A$ could be readily detected, whereas SCN9A was low in hiPSC-CM cells (Figure 7(a)). We next determined if the absence of $S C N 9 A$ in hiPSC-CMs was due to our inability to detect SCN9A message. In the next series of experiments, we transfected both SCN5A and SCN9A into TSA201 at a 1:1 molar ratio and probed for message. Results show that both $S C N 5 A$ and SCN9A message could be found in transfected TSA201 cells (Figure 7(b)) indicating that SCN9A can be readily detected under our experimental conditions and that the message of SCN9A is very low or absent in hiPSC-CMs. Similarly, we probed for PXDNL and its homolog, PXDN, in both WT and MMRL1126 hiPSC-CM. Results show that PXDN message was detected in hiPSC-CM, but that PXDNL expression was very low in hiPSC-CMs, as similarly reported [29].

Since PXDNL expression is very low in hiPSC-CMs, it seemed likely that any potential interaction of the protein with ion channels may not be observed in hiPSC-CM due to the low levels of PXDNL. In the first set of experiments, we transiently transfected either WT or R391Q PXDNL together with SCN5A. Similar to data shown in Figure 5, $I_{\mathrm{Na}}$ was activated by a series of depolarizing pulses from -80 to $+45 \mathrm{mV}$ in $5 \mathrm{mV}$ increments. The current-voltage relation showed $I_{\mathrm{Na}}$ was significantly reduced for R391Q compared to WT (Figure $8(\mathrm{~b}), p<0.05)$. In the next set of experiments, we transiently transfected either WT or R391Q PXDNL together with KCND3 and recorded $I_{\text {to }} . I_{\text {to }}$ was activated by a series of depolarizing pulses from -60 to $+50 \mathrm{mV}$ in $10 \mathrm{mV}$ increments (Figure 8(c)). The current-voltage relation showed that peak $I_{\text {to }}$ was significantly greater in R391Q mutant at potentials above $-20 \mathrm{mV}$ (Figure $8(\mathrm{~d}), p<0.05$ ).

The rise phase of the $\mathrm{Ca}^{2+}$ transient is governed by a combination of $\mathrm{Ca}^{2+}$ influx through $\mathrm{Ca}^{2+}$ channels and $\mathrm{Ca}^{2+}$ release from intracellular stores. Since the magnitude of the $\mathrm{Ca}^{2+}$ transient was smaller in hiPSC-CM derived from MMRL1126, we speculated that the mutation in FKBP1b indirectly affected the size of the $\mathrm{Ca}^{2+}$ current. In the next series of experiments, $I_{\mathrm{Ca}}$ was recorded under voltage-clamp conditions. Following a series of 3 prepulses (prepulses not shown) $I_{\mathrm{Ca}}$ was activated by a series of depolarizing pulses from -40 to $+50 \mathrm{mV}$ in $10 \mathrm{mV}$ increments. Figure 9(a) shows representative $I_{\mathrm{Ca}}$ traces from a control and patient-derived hiPSC-CM in response to the voltage clamp protocol. The threshold for $I_{\mathrm{Ca}}$ activated at $-30 \mathrm{mV}$ and peaked at $+10 \mathrm{mV}$. The current-voltage relation showed that $I_{\mathrm{Ca}}$ density was smaller in MMRL1126 hiPSC-CMs compared to control (Figure 9(b)). Finally, RNA expression of $\mathrm{Ca}^{2+}$ channel $\alpha$-subunits was examined by RT-PCR analysis. We examined both isoforms of L-type $\mathrm{Ca}^{2+}$ channels (Cav1.2 and Cav1.3). Our results show the lower $I_{\mathrm{Ca}}$ density observed in MMRL1126 was paralleled by lower message of Cav1.2 and Cav1.3 (Figure 9(c)). 
TABLE 2: Calcium transient parameters from hiPSC-CMs.

\begin{tabular}{|c|c|c|c|c|}
\hline & $\begin{array}{l}\text { Spontaneous cycle } \\
\text { length }\end{array}$ & $\begin{array}{c}\text { Number with regular spontaneous cycle } \\
\text { length }\end{array}$ & $\begin{array}{l}\% \text { with regular spontaneous cycle } \\
\text { length }\end{array}$ & $\left(F-F_{0}\right) / F_{0}$ \\
\hline Control & $\begin{array}{c}1857.8 \pm 56.1 \mathrm{~ms} \\
(n=102)\end{array}$ & $102 / 102$ & $100 \%$ & $\begin{array}{c}3.83 \pm 0.29 \\
(n=102)\end{array}$ \\
\hline $\begin{array}{l}\text { MMRL } \\
1239\end{array}$ & $\begin{array}{l}2310.8 \pm 136.2 \mathrm{~ms} \\
\quad(n=70)\end{array}$ & $70 / 75$ & $93.3 \%$ & $\begin{array}{l}3.78 \pm 0.24 \\
(n=93)\end{array}$ \\
\hline $\begin{array}{l}\text { MMRL } \\
1126\end{array}$ & $\begin{array}{c}2802.2 \pm 186.9 \mathrm{~ms} \\
(n=59)\end{array}$ & $59 / 93$ & $63.4 \%$ & $\begin{array}{c}2.03 \pm 0.29 \\
(n=93)\end{array}$ \\
\hline
\end{tabular}
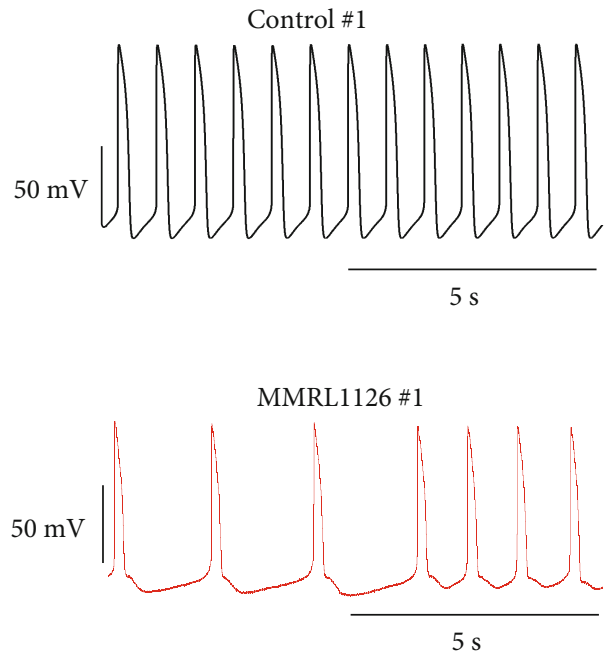

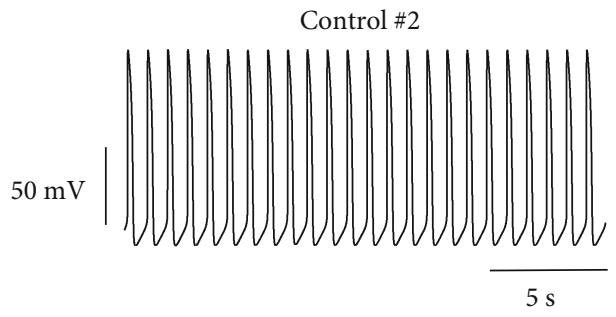

(a)

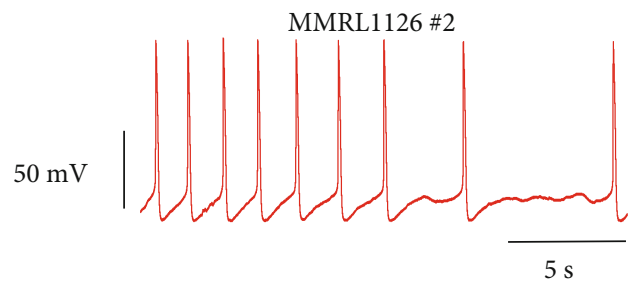

(b)

FIGURE 5: Action potential (AP) recordings obtained from WT and MMRL1126 monolayers. WT hiPSC-CMs were more hyperpolarized and had a larger $V_{\max }$. In addition, hiPSC-CMs from MMRL1127 exhibited slower spontaneous activity, and some showed episodic activity.

TABLE 3: Electrophysiological parameters.

\begin{tabular}{lcccc}
\hline & APD50 & APD90 & $V_{\max }$ & Maximim diastolic membrane potential \\
\hline Control & $168.0 \pm 12.5 \mathrm{~ms}(n=25)$ & $217.4 \pm 13.9 \mathrm{~ms}(n=25)$ & $38.5 \pm 2.4 \mathrm{~V} / \mathrm{s}(n=25)$ & $-68.7 \pm 1.1 \mathrm{mV}(n=25)$ \\
MMRL1126 & $119.2 \pm 7.6 \mathrm{~ms}(n=13)$ & $163.0 \pm 9.4 \mathrm{~ms}(n=13)$ & $17.3 \pm 1.6 \mathrm{~V} / \mathrm{s}(n=13)$ & $-57.8 \pm 2.1 \mathrm{mV}(n=13)$ \\
\hline
\end{tabular}

5 monolayers from MMRL1126 exhibited continuous spontaneous activity; 8 monolayers from MMRL1126 exhibited episodic activity.

\section{Discussion}

In this study, we identified a patient (MMRL 1126) who initially presented with signs of Early Repolarization Syndrome and monomorphic VT when infused with dobutamine. His syncope was clearly related to his sustained monomorphic VT. Following ablation of potential arrhythmic foci, patient subsequently developed right bundle branch block. Ajmaline challenge unmasked a BrS ECG. Genetic analysis of the patient showed the presence of mutations in three genes, FKBP1b, SCN9A, and PXDNL, none of which had previously been shown to be associated with ERS, BrS, or RBBB. All family members underwent genetic testing, and pedigree analysis revealed that the proband inherited all 3 mutations from the parents. Carriers of the single or double mutation, both parents and sister, did not display any overt clinical phenotype. In addition,
hiPSC-CMs derived from the unaffected father (MMRL 1239) also did not show any abnormalities in terms of beating frequency and $\mathrm{Ca}^{2+}$ transient magnitude. ERS and $\mathrm{BrS}$ are often referred to as J-wave syndromes [35] as they exhibit many similarities and both predispose to life-threatening ventricular arrhythmias. Development of these arrhythmias has previously been associated with alterations in $I_{\mathrm{Na}}, I_{\mathrm{Ca}}, I_{\mathrm{K}, \mathrm{ATP}}$, and $I_{\text {to }}$ due to ion channel mutations $[19,36,37]$. In our study, patch-clamp recordings of $I_{\mathrm{Ca}}$ in hiPSC-CMs derived from the patient revealed that $I_{\mathrm{Ca}}$ was reduced compared to WT hiPSC$\mathrm{CMs}$ suggesting that a reduction in this current may contribute to the mixed clinical phenotype.

Surprisingly, the ECG abnormalities manifested only in the individual who carried 3 mutations. It is unclear which gene(s) played the greater role in the development of the phenotype, since all genes identified in the proband have not 


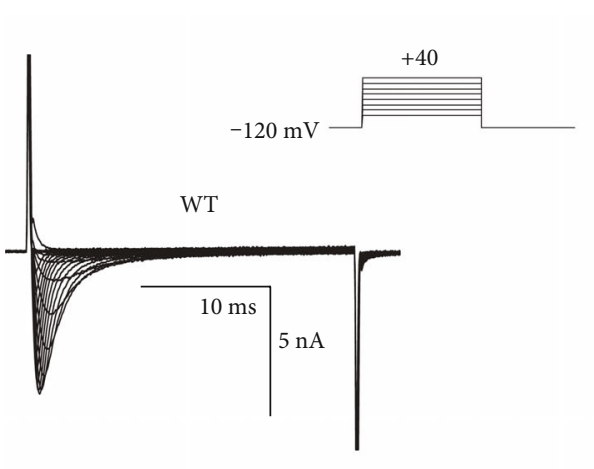

(a)

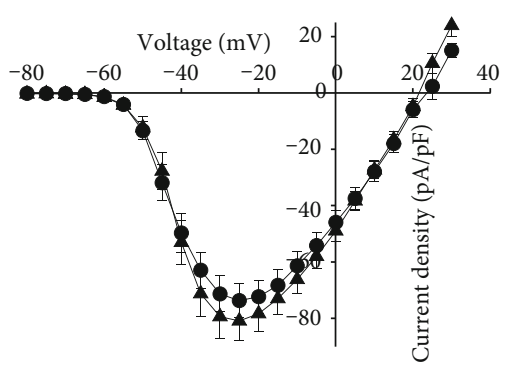

(c)

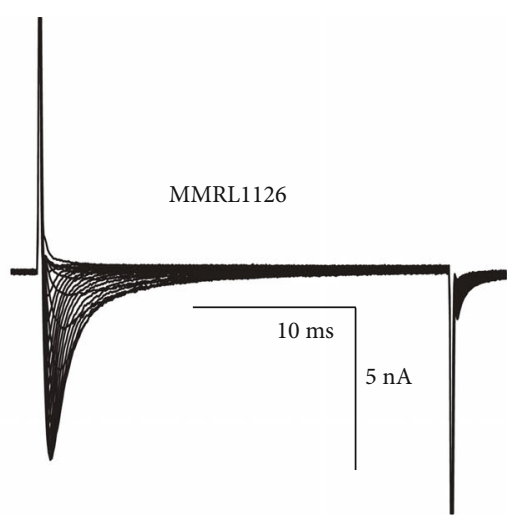

(b)

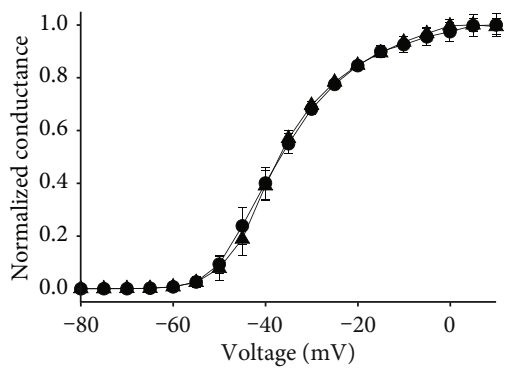

(d)

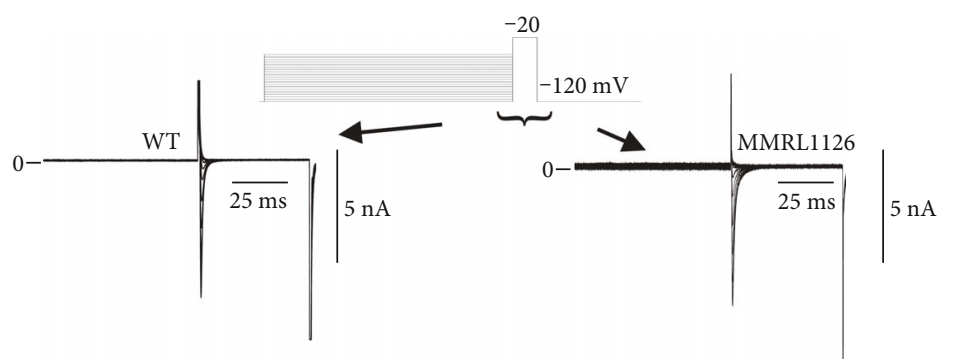

(e)

(f)

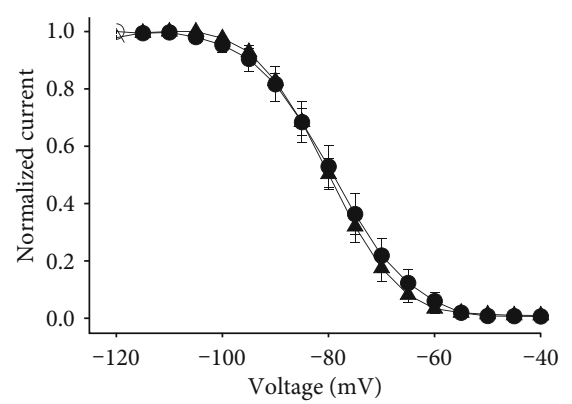

(g)

- $\mathrm{WT}(n=13)$

MMRL1126 $(n=14)$

FIGURE 6: Representative $I_{\mathrm{Na}}$ recordings from a WT (a) and MMRL1126 hiPSC-CM (b). Current recordings were obtained at test potentials between -80 and $25 \mathrm{mV}$ in $5 \mathrm{mV}$ increments. The holding potential was $-120 \mathrm{mV}$. (c) I-V relation for WT $(n=13)$ and MMRL1126 ( $n=14)$ showing no difference in $I_{\mathrm{Na}}$ magnitude. (d) Steady-state activation relation for WT and MMRL1126 hiPSC-CM cells. Chord conductance was determined using the ratio of current to the electromotive potential for the cells shown in (a) and (b). Data were normalized and plotted against their test potential. Representative steady-state inactivation recordings from a WT (e) and MMRL1126 hiPSC-CM (f). The voltage clamp protocol is shown at the top of the figure. Peak current was normalized to their respective maximum values and plotted against the conditioning potential. The mean data for the steady-state inactivation relation is shown (g). 


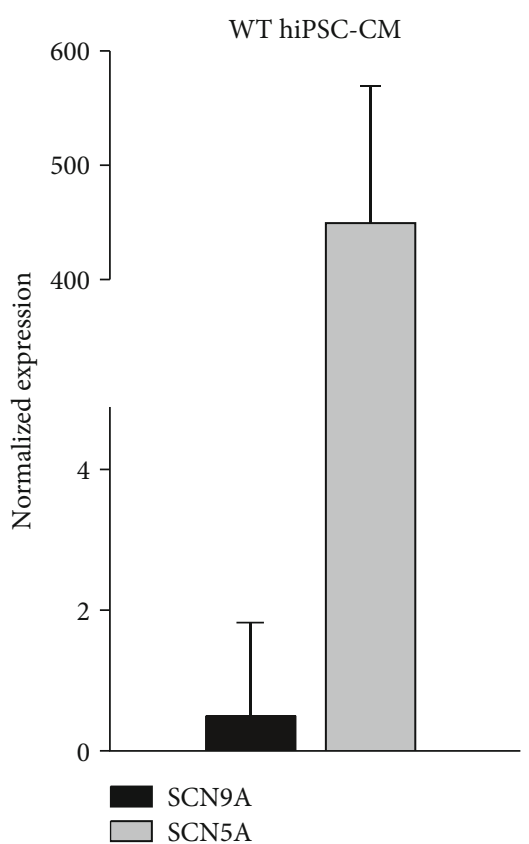

(a)

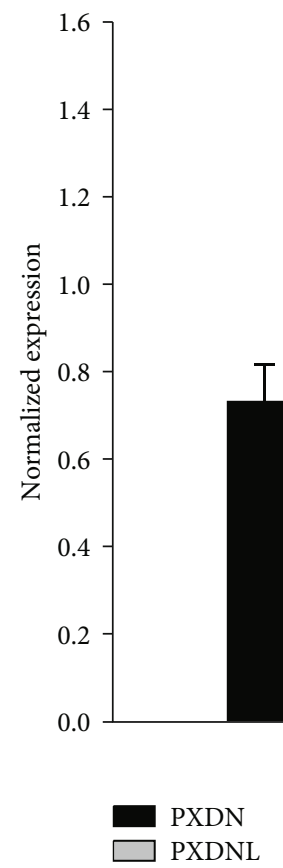

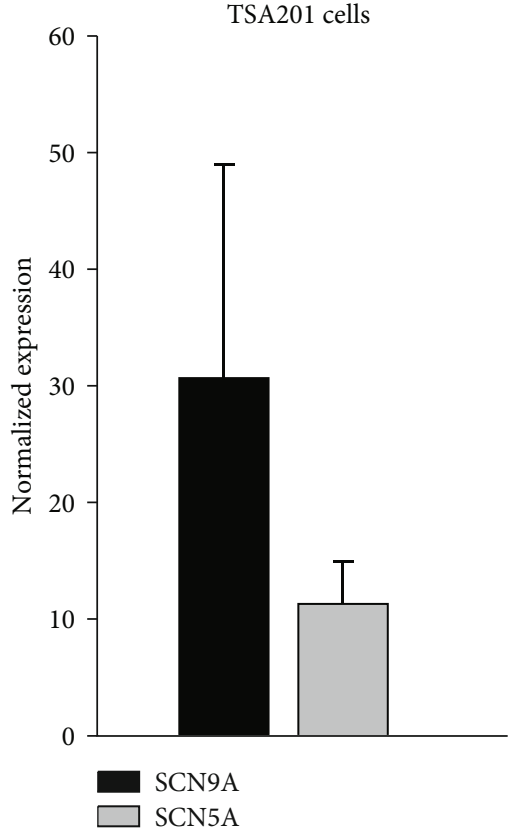

(b)

(c)

FIGURE 7: RT-qPCR analysis of SCN9A and SCN5A in hiPSC-CMs (normalized to GAPDH). SCN5A message was highly expressed, whereas levels of SCN9A were negligible in the hiPSC-CMs (a). RT-qPCR analysis of SCN9A and SCN5A in TSA201 cells transfected with SCN5A and SCN9A plasmid at a 1:1 molar ratio. SCN9A message could readily be detected in transfected TSA201 cells (b). RT-qPCR analysis of PXDN and PXDNL in hiPSC-CMs (c). PXDNL was expressed at low levels in both WT and MMRL1126 hiPSC-CMs.

previously been shown to be associated with any cardiac arrhythmia syndrome disease. The PXDNL gene encodes for a peroxidasin-like protein which is expressed in the heart [29]. Interestingly, the PXDNL proteins encoded by primate genomes appear to lack peroxidase activity suggesting no apparent functional role of the PXDNL protein in human heart [29]. In hiPSC myocytes, PXDNL message and protein were expressed at low levels [29], an observation confirmed in our study. In contrast, adult human ventricular sections showed staining mainly at the intercalated discs with some staining at the lateral surface [29] demonstrating that PXDNL protein is expressed at low levels in neonatal tissue but increase with age and maturity. Whether the presence of this protein at the intercalated disc affects cardiac conduction in adult ventricle and contributes to either the BrS or ERS phenotype remains to be determined. It is worth noting 

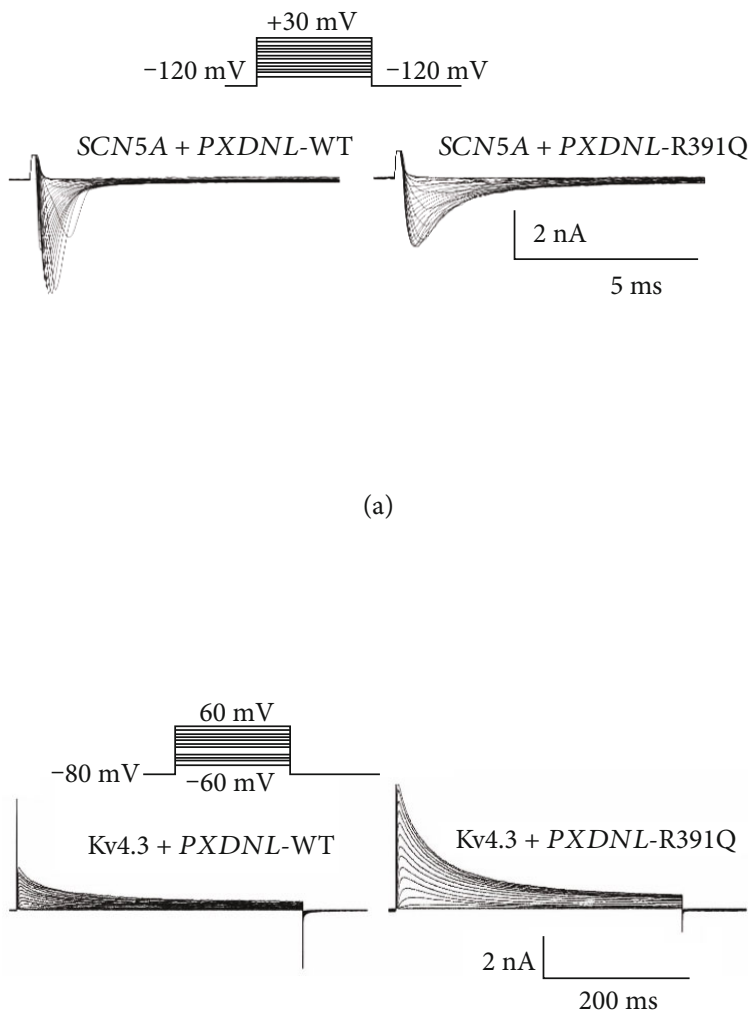

(c)

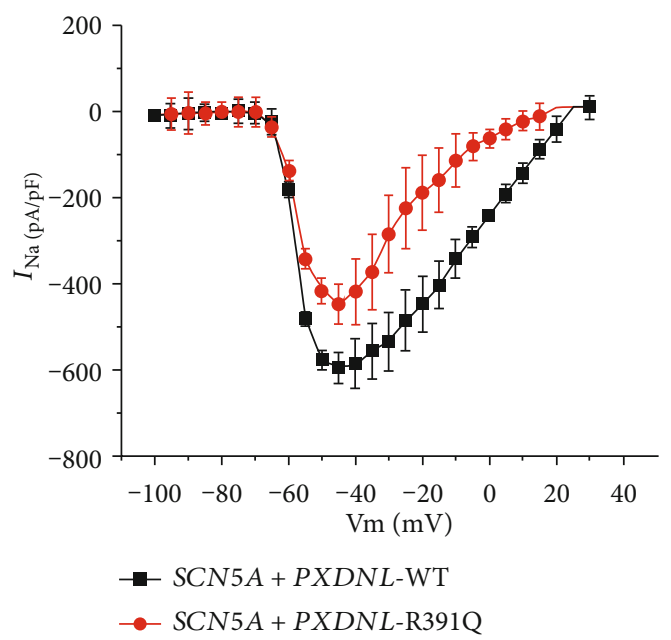

(b)

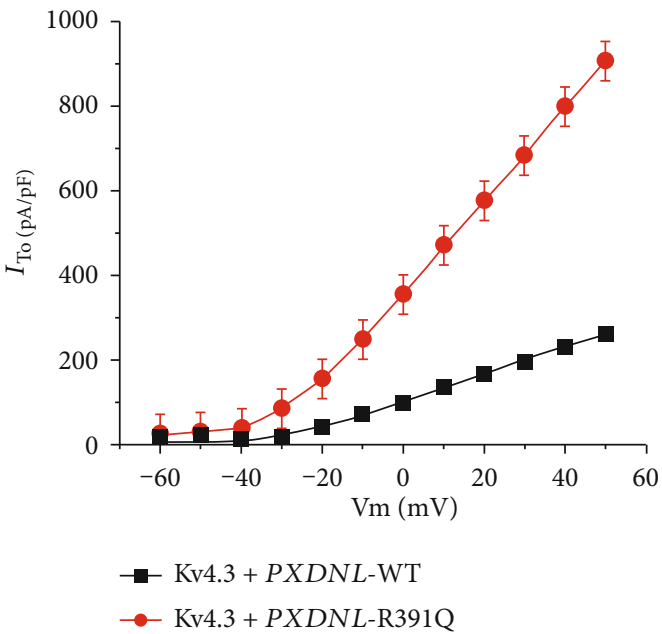

(d)

FIgURE 8: (a) Representative $I_{\mathrm{Na}}$ recordings from a WT and R391Q mutant in PXDNL cotransfected with SCN5A. I-V relation for WT and R391Q mutant in PXDNL cotransfected with SCN5A. The I-V relation was generated using same voltage clamp protocol described in Figure 5. A small reduction in $I_{\mathrm{Na}}$ density was noted with R391Q mutation. Representative $I_{\text {to }}$ recordings from a WT and R391Q (a) mutant in PXDNL cotransfected with KCND3. Current recordings were obtained at test potentials between -60 and $50 \mathrm{mV}$ from a holding potential was $-80 \mathrm{mV}$. (c) I-V relation for WT and R391Q showing a significant difference in $\mathrm{I}_{\text {to }}$ magnitude at potentials above $-20 \mathrm{mV}$.

that we have screened 403 patients using NextGen sequencing and have discovered 67 individuals with PXDNL variations at a minor allele frequency of $3 \%$ or below (75 variations in total as some individuals had multiple variations). The high association of PXDNL variations in patients exhibiting cardiac arrhythmias coupled with the strong expression of the protein in the ventricles (specifically the intercalated discs) suggests a link between this protein and the development of cardiac arrhythmias.

The role of the SCN9A in cardiac tissue is unclear but a similar disconnection between expression and functional role of SCN10A in cardiac tissue has been described [38-40]. In our study, PCR analysis of SCN9A message was performed in WT hiPSC-CM and revealed that message was very low in hiPSC-CMs (Figure 6). Consistent with our observations, no cardiac abnormalities have been identified in patients with either a gain [32] or complete loss of function of Nav1.7 [31]. To our knowledge, one study has noted the presence of Nav1.7 in hiPSC-CMs [41]. Although previous studies have demonstrated a role for "brain" type $\mathrm{Na}^{+}$channels in adult heart [30, 42, 43], our results suggest that Nav1.7 message is very low in hiPSC-CMs suggesting a minor role of "brain" type Nav1.7 in hiPSC-CMs. Whether Nav1.7 protein is expressed and has a functional role in adult human heart is unclear (for review see Zimmer et al. [44]).

The role of the FKBP1B mutation in MMRL1126 is readily apparent in our study. We were able to detect FKBP1b message in hiPSC-CM, and functional analysis of the calcium transient showed dramatic differences in fluorescence intensity and spontaneous beating rate compared to WT hiPSCCMs. Patch clamp recordings of $I_{\mathrm{Ca}}$ in hiPSC-CMs derived from MMRL1126 showed about a 50\% amplitude reduction 


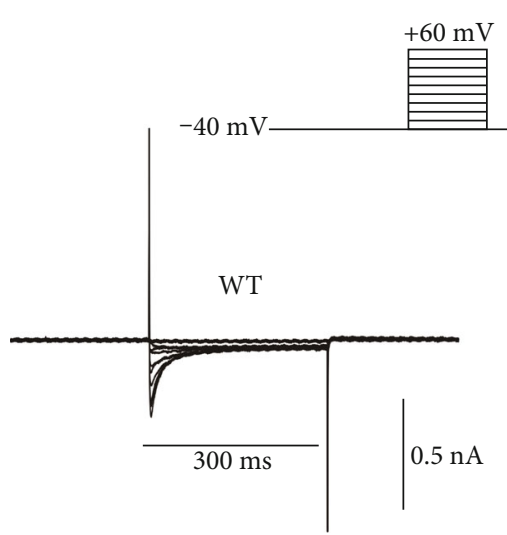

(a)

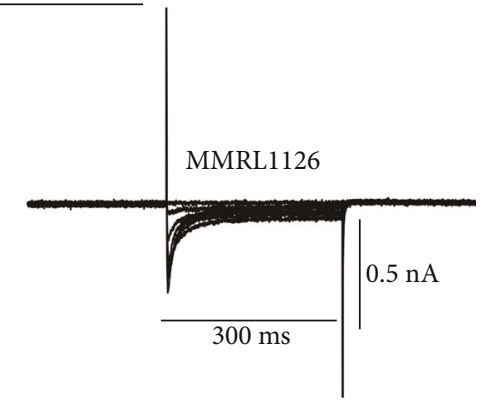

(b)

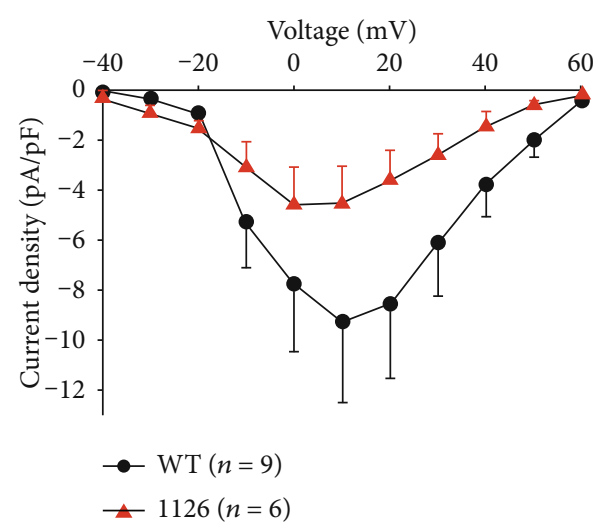

(c)

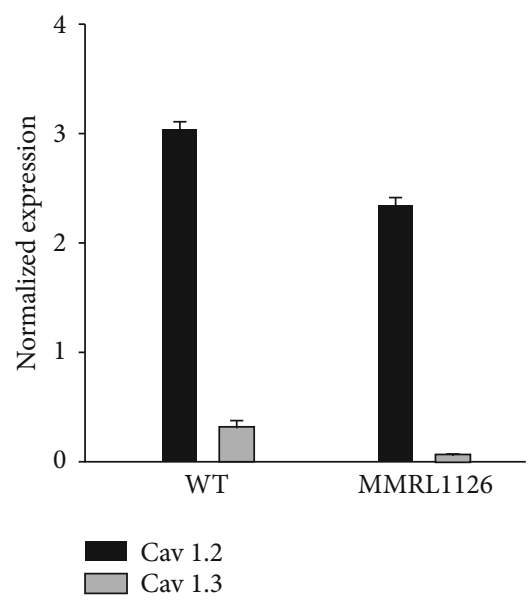

(d)

FIGURE 9: Representative traces showing $I_{\mathrm{Ca}}$ recorded from (a) WT and (b) MMRL1126. $\mathrm{Ca}^{2+}$ currents were recorded during a 300 ms step depolarization from -40 to $+50 \mathrm{mV}$ in $10 \mathrm{mV}$ increments. (c) Current-voltage relationship for $I_{\mathrm{Ca}}$. (d) RT-PCR showing a reduction of Cav1.2 and Cav1.3 message in MMRL1126.

in peak $\mathrm{Ca}^{+}$current compared to WT. Previous studies have demonstrated that a reduction in peak $I_{\mathrm{Ca}}$ secondary to mutation in Cav1.2 channel subunits can contribute to both the BrS [45, 46] or ERS phenotype [47]. It is unclear if the episodes of syncope and palpitations in this patient are related to ERS, BrS, or RBBB. Using hiPSC-CMs from the proband, we demonstrate that the $\mathrm{Ca}^{2+}$ transient is altered in spontaneously active hiPSC-CMs. Since we demonstrate that hiPSC-CMs exhibited altered calcium cycling, a similar situation may be occurring in the patient, likely explaining the syncope following dobutamine infusion.

The manifestation of ERS and BrS (J-wave syndromes) is due to an imbalance in depolarizing and/or repolarizing currents in the early parts of the cardiac action potential. A decrease in inward $I_{\mathrm{Na}}$ or $I_{\mathrm{Ca}}$ will accentuate the spike-anddome morphology of the action potential mainly in the 
epicardium (epi) generating a transmural voltage gradient that leads to the characteristic ECG changes $[13,14]$. In our study, the mutation in $F K B P 1 B$ resulted in alterations in intracellular $\mathrm{Ca}^{2+}$ cycling as well as reduced $I_{\mathrm{Ca}}$ in hiPSCCMs derived from the patient when compared to WT hiPSC-CMs. In addition, the PXDNL mutation resulted in a reduced $I_{\mathrm{Na}}$ when compared with WT PXDNL. Together, mutations in FKBP1b, SCN9A, and PXDNL have a multifactorial effect on the reduction of the two currents resulting in the clinical phenotype. The PXDNL variant in addition to producing a loss of function of $I_{\mathrm{Na}}$ caused a significant increase in $I_{\text {to }}$, which may have added contributions with the reductions in $I_{\mathrm{Na}}$ and $I_{\mathrm{Ca}}$.

The modelling of complex arrhythmia syndromes in hiPSC-CMs has been performed but with variable results, likely due to the immaturity of pluripotent cells as well as the methods used in the various laboratories to derive hiPSC-CMs. For example, several studies have attempted to model BrS in patient-derived hiPSC-CMs but with limited success due largely to the immaturity of the transient outward current in hiPSC-CM [48]. Studies have suggested that ion channel analysis in hiPSC myocytes are superior compared to mammalian expression systems due to the presence of auxiliary subunits [49]. However, other studies have reported no differences in the electrophysiological characteristics (assessed by action potential and microelectrode array recordings) between stem cell-derived cardiomyocytes from a Brugada Syndrome patient and those derived from controls [50]. These observations suggest that hiPSC-CMs are not effective in recapitulating complex phenotypes (such as $\mathrm{BrS}$ ) where factors such as fibrosis, cellular coupling/uncoupling, and epi- to endorepolarization differences are important.

\section{Conclusions}

Many ventricular arrhythmia syndromes have been linked to mutations in SCN5A [51, 52], CACNA1C, CACNB2, and CACNA2D1 [45-47]. The latter genes form the L-type calcium channel complex and mutations in these genes produced a reduction in the size of $I_{\mathrm{Ca}}$ resulting in BrS/ERS phenotypes. Our patch clamp studies showed that $I_{\mathrm{Ca}}$ was also reduced in hiPSC-CM from MMRL1126. Although 3 family members carried either one or two mutations, none of them has developed arrhythmias to date. In contrast, the proband who inherited all 3 mutations developed palpitations, syncope, and displayed ERS and BrS phenotypes. Using hiPSC myocytes derived from the patient, we demonstrate a clear alteration in several physiological parameters such as reduced ion current density and altered excitation contraction coupling when compared to normal hiPSC myocytes. Consistent with our findings in this study, certain arrhythmia syndromes, such as BrS and ERS, are polygenic in nature [53] with contributions from two or more genetic variants. The results of our study highlight the complexed interaction between genetic mutations and phenotypic expression and demonstrate the effectiveness of hiPSC myocytes manufactured from the patient to study the phenotypic changes.

\section{Data Availability}

The data that support the findings of this study are available on request from the corresponding author at jcordeiro@mmri.edu.

\section{Disclosure}

HBM, MS, and DH are co-first authors.

\section{Conflicts of Interest}

The authors report no relationships that could be construed as a conflict of interest.

\section{Acknowledgments}

Drs. Antzelevitch and Barajas acknowledge support from NHLBI (HL47678, HL138103, and HL152201), the Martha and Wistar Morris Fund, and the W/W. Smith Trust. Dr. Hu acknowledges support by the National Natural Science Foundation Project of China (Grant no. 81670304), distinguished professor of Chutian Scholar Program of Hubei Province. Dr. Cordeiro acknowledges support from HL145530-01A1 and the Free and Accepted Masons of New York, Florida, Massachusetts, Connecticut, Maryland, Wisconsin, Washington, and Rhode Island.

\section{References}

[1] J. C. Wu, P. Garg, Y. Yoshida et al., "Towards precision medicine with human iPSCs for cardiac channelopathies," Circulation Research, vol. 125, no. 6, pp. 653-658, 2019.

[2] M. X. Doss, J. M. Di Diego, R. J. Goodrow et al., "Maximum diastolic potential of human induced pluripotent stem cellderived cardiomyocytes depends critically on $\mathrm{I}_{\mathrm{Kr}}$," PLoS One, vol. 7, no. 7, article e40288, 2012.

[3] G. C. Bett, A. D. Kaplan, A. Lis et al., "Electronic "expression" of the inward rectifier in cardiocytes derived from humaninduced pluripotent stem cells," Heart Rhythm, vol. 10, no. 12, pp. 1903-1910, 2013.

[4] S. G. Priori, A. A. Wilde, M. Horie et al., "Executive summary: HRS/EHRA/APHRS expert consensus statement on the diagnosis and management of patients with inherited primary arrhythmia syndromes," Heart Rhythm, vol. 10, no. 12, pp. 1932-1963, 2013.

[5] O. Campuzano, G. Sarquella-Brugada, S. Cesar, E. Arbelo, J. Brugada, and R. Brugada, "Recent advances in short QT syndrome," Frontiers in Cardiovascular Medicine, vol. 5, 2018.

[6] P. Brugada and J. Brugada, "Right bundle branch block, persistent ST segment elevation and sudden cardiac death: a distinct clinical and electrocardiographic syndrome: a multicenter report," Journal of the American College of Cardiology, vol. 20, no. 6, pp. 1391-1396, 1992.

[7] J. M. Di Diego and C. Antzelevitch, "J wave syndromes as a cause of malignant cardiac arrhythmias," Pacing and Clinical Electrophysiology, vol. 41, no. 7, pp. 684-699, 2018.

[8] D. Hu, H. Barajas-Martinez, E. Burashnikov et al., "A mutation in the beta 3 subunit of the cardiac sodium channel associated with Brugada ECG phenotype," Heart Rhythm, vol. 2, no. 3, pp. 270-278, 2009. 
[9] C. Antzelevitch, "J wave syndromes: molecular and cellular mechanisms," Journal of Electrocardiology, vol. 46, no. 6, pp. 510-518, 2013.

[10] E. Burashnikov, R. Pfeiffer, H. Barajas-Martinez et al., "Mutations in the cardiac L-type calcium channel associated with inherited sudden cardiac death Syndromes," Heart Rhythm, vol. 7, no. 11, 2010.

[11] P. J. Mohler, J.-J. Schott, A. O. Gramolini et al., “Ankyrin-B mutation causes type 4 long-QT cardiac arrhythmia and sudden cardiac death," Nature, vol. 421, no. 6923, pp. 634-639, 2003.

[12] P. J. Mohler and V. Bennett, "Ankyrin-based cardiac arrhythmias: a new class of channelopathies due to loss of cellular targeting," Current Opinion in Cardiology, vol. 20, no. 3, pp. 189-193, 2005.

[13] K. Calloe, J. M. Cordeiro, J. M. Di Diego et al., “A transient outward potassium current activator recapitulates the electrocardiographic manifestations of Brugada syndrome," Cardiovascular Research, vol. 81, no. 4, pp. 686-694, 2009.

[14] I. Koncz, Z. Gurabi, B. Patocskai et al., "Mechanisms underlying the development of the electrocardiographic and arrhythmic manifestations of early repolarization syndrome," Journal of Molecular and Cellular Cardiology, vol. 68, pp. 2028,2014

[15] S. E. Lehnart, C. Terrenoire, S. Reiken et al., "Stabilization of cardiac ryanodine receptor prevents intracellular calcium leak and arrhythmias," Proceedings of the National Academy of Sciences of the United States of America, vol. 103, no. 20, pp. 7906-7910, 2006.

[16] M. E. Curran, I. Splawski, K. W. Timothy, G. M. Vincent, E. D. Green, and M. T. Keating, "A molecular basis for cardiac arrhythmia: HERG mutations cause long QT syndrome," Cell, vol. 80, no. 5, pp. 795-803, 1995.

[17] R. Brugada, K. Hong, R. Dumaine et al., "Sudden death associated with short-QT syndrome linked to mutations in HERG," Circulation, vol. 109, no. 1, pp. 30-35, 2004.

[18] D. Hu, Y. Li, J. Zhang et al., "The phenotypic spectrum of a mutation hotspot responsible for the short QT syndrome," JACC: Clinical Electrophysiology, vol. 3, no. 7, pp. 727-743, 2017.

[19] M. Haissaguerre, S. Chatel, F. Sacher et al., "Ventricular fibrillation with prominent early repolarization associated with a rare variant of $\mathrm{KCNJ} 8 / \mathrm{K}_{\mathrm{ATP}}$ channel," Journal of cardiovascular electrophysiology, vol. 20, no. 1, pp. 93-98, 2009.

[20] D. Hu, H. Barajas-Martinez, A. Terzic et al., " $A B C C 9$ is a novel Brugada and early repolarization syndrome susceptibility gene," International Journal of Cardiology, vol. 171, no. 3, pp. 431-442, 2014.

[21] J. A. Treat, R. J. Goodrow, C. T. Bot, R. J. Haedo, and J. M. Cordeiro, "Pharmacological enhancement of repolarization reserve in human induced pluripotent stem cells derived cardiomyocytes," Biochemical Pharmacology, vol. 169, article 113608, 2019.

[22] J. M. Cordeiro, J. H. Bridge, and K. W. Spitzer, "Early and delayed afterdepolarizations in rabbit heart Purkinje cells viewed by confocal microscopy," Cell Calcium, vol. 29, no. 5, pp. 289-297, 2001.

[23] J. M. Cordeiro, J. E. Malone, J. M. Di Diego et al., "Cellular and subcellular alternans in the canine left ventricle," American Journal of Physiology. Heart and Circulatory Physiology, vol. 293, no. 6, pp. H3506-H3516, 2007.
[24] H. M. Barajas-Martínez, D. Hu, J. M. Cordeiro et al., "Lidocaine-induced Brugada syndrome phenotype linked to a novel double mutation in the cardiac sodium channel," Circulation Research, vol. 103, no. 4, pp. 396-404, 2008.

[25] R. J. Goodrow Jr., S. Desai, J. A. Treat et al., "Biophysical comparison of sodium currents in native cardiac myocytes and human induced pluripotent stem cell-derived cardiomyocytes," Journal of Pharmacological and Toxicological Methods, vol. 90, pp. 19-30, 2018.

[26] K. Calloe, R. Goodrow, S. P. Olesen, C. Antzelevitch, and J. M. Cordeiro, "Tissue specific effects of acetylcholine in the canine heart," American Journal of Physiology. Heart and Circulatory Physiology, vol. 305, no. 1, pp. H66-H75, 2013.

[27] H. Barajas-Martinez, R. J. Goodrow, D. Hu et al., "Biophysical and molecular comparison of sodium current in cells isolated from canine atria and pulmonary vein," Pflügers Archiv - European Journal of Physiology, vol. 469, no. 5-6, pp. 703-712, 2017.

[28] S. Kaab, H. B. Nuss, N. Chiamvimonvat et al., "Ionic mechanism of action potential prolongation in ventricular myocytes from dogs with pacing-induced heart failure," Circulation research, vol. 78, no. 2, pp. 262-273, 1996.

[29] Z. Peterfi, Z. E. Toth, H. A. Kovacs et al., "Peroxidasin-like protein: a novel peroxidase homologue in the human heart," Cardiovascular Research, vol. 101, no. 3, pp. 393-399, 2014.

[30] V. Haufe, J. M. Cordeiro, T. Zimmer et al., "Contribution of neuronal sodium channels to the cardiac fast sodium current $I_{\mathrm{Na}}$ is greater in dog heart Purkinje fibers than in ventricles," Cardiovascular Research, vol. 65, no. 1, pp. 117-127, 2005.

[31] J. J. Cox, F. Reimann, A. K. Nicholas et al., "An SCN9A channelopathy causes congenital inability to experience pain," Nature, vol. 444, no. 7121, pp. 894-898, 2006.

[32] J. W. Theile, B. W. Jarecki, A. D. Piekarz, and T. R. Cummins, "Nav1.7 mutations associated with paroxysmal extreme pain disorder, but not erythromelalgia, enhance Navbeta4 peptide-mediated resurgent sodium currents," The Journal of physiology, vol. 589, no. 3, pp. 597-608, 2011.

[33] S. K. Maier, R. E. Westenbroek, K. A. McCormick, R. Curtis, T. Scheuer, and W. A. Catterall, "Distinct subcellular localization of different sodium channel $\mathrm{a}$ and $\mathrm{b}$ subunits in single ventricular myocytes from mouse heart," Circulation, vol. 109, no. 11, pp. 1421-1427, 2004.

[34] V. Haufe, J. A. Camacho, R. Dumaine et al., "Expression pattern of neuronal and skeletal muscle voltage-gated $\mathrm{Na}^{+}$ channels in the developing mouse heart," The Journal of Physiology, vol. 564, no. 3, pp. 683-696, 2005.

[35] C. Antzelevitch and G. X. Yan, "J-wave syndromes. from cell to bedside," Journal of electrocardiology, vol. 44, no. 6, pp. 656661, 2011.

[36] A. Medeiros-Domingo, B. H. Tan, L. Crotti et al., "Gain-offunction mutation S422L in the KCNJ8-encoded cardiac $\mathrm{K}_{\mathrm{ATP}}$ channel Kir6.1 as a pathogenic substrate for J-wave syndromes," Heart Rhythm, vol. 7, no. 10, pp. 1466-1471, 2010.

[37] H. Barajas-Martinez, D. Hu, T. Ferrer et al., "Molecular genomic and functional association of Brugada and early repolarization syndromes with S422L missense mutation in KCNJ8," Circulation, vol. 122, article A20293, 2010.

[38] D. Hu, H. Barajas-Martinez, R. Pfeiffer et al., "Mutations in $S C N 10 A$ are responsible for a large fraction of cases of Brugada syndrome," Journal of the American College of Cardiology, vol. 64, no. 1, pp. 66-79, 2014. 
[39] M. van den Boogaard, S. Smemo, O. Burnicka-Turek et al., "A common genetic variant within SCN10A modulates cardiac SCN5A expression," The Journal of Clinical Investigation, vol. 124, no. 4, pp. 1844-1852, 2014.

[40] A. O. Verkerk, C. A. Remme, C. A. Schumacher et al., "Functional Nav1.8 channels in intracardiac neurons: the link between SCN10A and cardiac electrophysiology," Circulation research, vol. 111, no. 3, pp. 333-343, 2012.

[41] A. Moreau, A. Mercier, O. Theriault et al., "Biophysical, molecular, and pharmacological characterization of voltagedependent sodium channels from induced pluripotent stem cell-derived cardiomyocytes," Canadian Journal of Cardiology, vol. 33, no. 2, pp. 269-278, 2017.

[42] S. K. Maier, R. E. Westenbroek, K. A. Schenkman, E. O. Feigl, T. Scheuer, and W. A. Catterall, "An unexpected role for braintype sodium channels in coupling of cell surface depolarization to contraction in the heart," Proceedings of the National Academy of Sciences of teh Unnited States of America, vol. 99, no. 6, pp. 4073-4078, 2002.

[43] M. Biet, H. Barajas-Martinez, A. T. Ton, J. F. Delabre, N. Morin, and R. Dumaine, "About half of the late sodium current in cardiac myocytes from dog ventricle is due to noncardiac-type $\mathrm{Na}^{+}$channels," Journal of Molecular and Cellular Cardiology, vol. 53, no. 5, pp. 593-598, 2012.

[44] T. Zimmer, V. Haufe, and S. Blechschmidt, "Voltage-gated sodium channels in the mammalian heart," Global Cardiology Science and Practice, vol. 2014, no. 4, pp. 449-463, 2014.

[45] C. Antzelevitch, G. D. Pollevick, J. M. Cordeiro et al., "Loss-offunction mutations in the cardiac calcium channel underlie a new clinical entity characterized by ST-segment elevation, short QT intervals, and sudden cardiac death," Circulation, vol. 115, no. 4, pp. 442-449, 2007.

[46] J. M. Cordeiro, M. Marieb, R. Pfeiffer, K. Calloe, E. Burashnikov, and C. Antzelevitch, "Accelerated inactivation of the L-type calcium due to a mutation in CACNB2b due to a mutation in CACNB2b underlies Brugada syndrome," Journal of Molecular and Cellular Cardiology, vol. 46, no. 5, supplement 18, pp. 695-703, 2009.

[47] E. Burashnikov, R. Pfeiffer, H. Barajas-Martinez et al., "Mutations in the cardiac L-type calcium channel associated J wave sydnrome and sudden cardiac death," Heart Rhythm, vol. 7, no. 12, pp. 1872-1882, 2010.

[48] J. M. Cordeiro, V. V. Nesterenko, S. Sicouri et al., "Identification and characterization of a transient outward $\mathrm{K}^{+}$current in human induced pluripotent stem cell-derived cardiomyocytes," Journal of Molecular and Cellular Cardiology, vol. 60, no. 1, pp. 36-46, 2013.

[49] E. Selga, F. Sendfeld, R. Martinez-Moreno et al., "Sodium channel current loss of function in induced pluripotent stem cell-derived cardiomyocytes from a Brugada syndrome patient," Journal of Molecular and Cellular Cardiology, vol. 114, pp. 10-19, 2018.

[50] C. C. Veerman, I. Mengarelli, K. Guan et al., "hiPSC-derived cardiomyocytes from Brugada Syndrome patients without identified mutations do not exhibit clear cellular electrophysiological abnormalities," Scientific reports, vol. 6, no. 1, article 30967, 2016.

[51] J. M. Cordeiro, H. Barajas-Martinez, K. Hong et al., "Compound heterozygous mutations $\mathrm{P} 336 \mathrm{~L}$ and $11660 \mathrm{~V}$ in the human cardiac sodium channel associated with the Brugada syndrome," Circulation, vol. 114, no. 19, pp. 2026-2033, 2006.
[52] H. Watanabe, A. Nogami, K. Ohkubo et al., "Electrocardiographic characteristics and SCN5A mutations in idiopathic ventricular fibrillation associated with early repolarization," Circulation. Arrhythmia and Electrophysiology, vol. 4, no. 6, pp. 874-881, 2011.

[53] M. Cerrone, C. A. Remme, R. Tadros, C. R. Bezzina, and M. Delmar, "Beyond the one gene-one disease paradigm: complex genetics and pleiotropy in inheritable cardiac disorders," Circulation, vol. 140, no. 7, pp. 595-610, 2019. 